\title{
Quantidade de Matéria e Concentração
}

\author{
João Carlos de Andrade \\ Rogério Custodio * \\ roger@iqm.unicamp.br \\ Universidade Estadual de Campinas, Instituto de Química
}

Informações do Artigo

Histórico do Artigo

Criado em Outubro de 1997

Atualizado em Março de 2000
Resumo

O presente artigo apresenta as definições de quantidade de matéria, mol e concentração, além das várias unidades aceitas pelo Sistema Internacional para concentração.

\section{Palavras-Chaves \\ Massa molar \\ $\mathrm{Mol}$ \\ Concentração \\ Molaridade \\ Molalidade \\ Normalidade}

Quantidade de matéria e concentração

A unidade SI para quantidade de matéria é o mol (mole, em inglês). O mol é definido como "a quantidade de matéria de um sistema que contém tantas unidades elementares quantos forem os átomos contidos em $0,012 \mathrm{~kg}$ de Carbono-12”. Por esta definição, qualquer quantidade de matéria que contenha $6,022 \times 10^{23}$ entidades é um mol. Assim, pode-se ter $1 \mathrm{~mol}$ de átomos, de moléculas, de íons, de prótons, de elétrons, ou de outras partículas, etc. Havendo tantas possibilidades, a entidade em questão deve ser sempre claramente especificada.

A expressão correta para se referir à massa de uma porção de substância cuja quantidade de matéria é um mol é a massa molar $(M)$. A massa molar pode se referir às moléculas, elementos, íons, elétrons, etc. Exemplo: $M(\mathrm{KCl})=74,56 \mathrm{~g} / \mathrm{mol}, M(\mathrm{Cu})=63,54 \mathrm{~g} / \mathrm{mol} ; M(\mathrm{H})=$
$1,0074 \mathrm{~g} / \mathrm{mol} ; M\left(\mathrm{Cl}_{2}\right)=70,916 \mathrm{~g} / \mathrm{mol}$.

O nome e o símbolo da unidade mol são idênticos. $\mathrm{O}$ plural do nome, muito usado no Brasil, é mols e não moles, analogamente a becquerels, henrys, pascals, etc, embora esses plurais não se ajustem às normas gramaticais da língua portuguesa.Entretanto, é imortante lembrar que o símbolo das unidades não mudam no plural (ex: $z=1,3$ $\mathrm{mol})$.

O emprego desta definição de mol tornou obsoletos e em desuso diversos termos como número de mols, número de moléculas-grama, número de átomosgrama (todos substituídos por quantidade de matéria); peso atômico e peso molecular (substituídos por massa molar), e molaridade e normalidade (substituídos por concentração em quantidade de matéria ou, simplesmente, concentração). 
Os termos massa nuclídica, massa atômica e massa molecular, todos com símbolo $\mathrm{m}$, têm um significado diferente daquele muitas vezes utilizado no passado. Eles se referem à massa de um dado nuclídio, átomo, ou molécula (ou fórmula unitária). Sua unidade é a unidade unificada de massa atômica (u), definida como $1 \mathrm{u}=1 \mathrm{~g} /$ $\mathrm{N}_{\mathrm{o}}$, onde $\mathrm{N}_{\mathrm{o}}$ é o número de Avogadro (6,022 $\left.\times 10^{23}\right)$, pois a unidade grama é muito grande para expressar a massa nuclídica, atômica ou molecular. Exemplos: $\mathrm{m}(35 \mathrm{Cl})=$ $34,97 \mathrm{u}$ ou $5,807 \times 10^{-23} \mathrm{~g} ; \mathrm{m}(\mathrm{Cl})=35,45 \mathrm{u}$ ou $5,887 \mathrm{x}$ $10^{-23} \mathrm{~g} ; \mathrm{m}(\mathrm{NaCl})=58,44 \mathrm{u}$ ou $9,704 \times 10^{-23} \mathrm{~g}$.

As unidades SI para concentração, (C), são $\mathrm{mol} / \mathrm{m}^{3}$, quando a massa molar for conhecida, e $\mathrm{kg} / \mathrm{m}^{3}$, se não o for. Múltiplos das unidades SI são, naturalmente, aceitos: $\mathrm{mol} / \mathrm{dm}^{3}, \mathrm{mmol} / \mathrm{dm}^{3}, \mathrm{mg} / \mathrm{kg}$ etc. Os volumes de soluções podem também ser expressos em litros. Exemplos:

$$
\begin{gathered}
\mathrm{C}\left(\mathrm{H}_{2} \mathrm{SO}_{4}\right)=0,5 \mathrm{~mol} / \mathrm{dm}^{3} \\
\mathrm{C}\left(\mathrm{K}^{+}\right)=2,0 \mathrm{~mol} / \mathrm{L}
\end{gathered}
$$

As concentrações medidas em mol (ex.: mol/L) não devem ser denominadas por molar, pois este termo deve apenas ser empregado quando associado ao nome de uma grandeza extensiva (que depende do tamanho da amostra), dividida pela quantidade de matéria, o mol (exemplos: massa molar, volume molar etc.). Além disso, como a concentração pode ser entendida como uma constante de proporcionalidade que relaciona alguma grandeza do soluto (quantidade de matéria (mol) ou massa $(\mathrm{g})$ ) com o volume (ou massa) da solução,

$$
\begin{aligned}
\mathrm{n}_{\mathrm{S}} & =\mathrm{C}_{\mathrm{SOL}} \times \mathrm{V}_{\mathrm{SOL}} \\
\mathrm{m}_{\mathrm{S}} & =\mathrm{C}_{\mathrm{SOL}} \times \mathrm{V}_{\mathrm{SOL}}
\end{aligned}
$$

então $\mathrm{C}_{\mathrm{SOL}}$, que é uma constante de proporcionalidade, tem que ser expressa em mol/L ou em $\mathrm{g} / \mathrm{L}$, dependendo do caso. Assim, como esta constante tem dimensões definidas, deve-se evitar a referência desta grandeza como Molaridade.

Por outro lado, o termo Molalidade ( $\mathrm{mol} / \mathrm{kg}$ de solvente) é um termo aceitável, sendo preferido para medidas precisas, em condições não isotérmicas. Concentrações expressas em mol/massa também são úteis em determinações de concentração feitas em sólidos, tais como em solos:

$$
\mathrm{C}(\mathrm{P})=0,5 \mathrm{~mol} / \mathrm{kg}
$$

mas não devem ser confundidas com a molalidade.

O uso de porcentagem (\%) deve se restringir aos casos estritamente necessários e o uso de partes por mil, partes por milhão (ppm) e partes por bilhão (ppb) deve ser eliminado, pois estas não são unidades SI. O principal problema com estes termos é a ambigüidade, pois eles podem se referir a relações massa/massa, massa/volume, volume/volume ou volume/massa. Assim, se o seu uso for necessário, é preciso dar referência à relação de comparação (ex.: $2 \% \mathrm{~m} / V$ ). $\mathrm{O}$ uso de porcentagem é aceitável quando em eventos que não possam ser descritos com unidades SI ou então quando se trata de uma comparação fracional bem definida. Alguns dos casos possíveis são: coeficiente de variação, composição de produtos comerciais, umidade relativa e átomo-porcento de abundância de isótopos estáveis, etc.

Ainda como conseqüência da definição de mol, as expressões equivalente-grama (e), normal (N) e normalidade tornaram-se obsoletas e estão em desuso. Por definição, o equivalente de uma espécie X é aquela entidade que, para uma determinada reação, combinará com:

a. uma entidade de íons hidrogênio ionizáveis, nas reações que não envolvem mudança no número de oxidação, como nas reações ácido-base, ou

b. uma entidade de elétrons, nas reações de óxidoredução. Nestes casos, é preciso levar em conta o conceito de Fator de Equivalência.

Para melhor explicá-lo, considerar uma reação genérica $\mu_{\mathrm{A}} \mathrm{A}+\mu_{\mathrm{B}} \mathrm{B} \rightleftharpoons$ Produtos; que pode ser re-escrita como

$$
\mathrm{A}+\left(\mu_{\mathrm{B}} / \mu_{\mathrm{A}}\right) \mathrm{B} \rightleftharpoons \text { Produtos }
$$

onde $\mu_{\mathrm{A}} \geq \mu_{\mathrm{B}}$ e a razão $\left(\mu_{\mathrm{B}} / \mu_{\mathrm{A}}\right)=\mathrm{f}_{\text {eq }} \geq 1$, sendo $\mathrm{f}_{\text {eq }}$ o Fator de Equivalência. Isto significa que uma entidade da espécie A será "equivalente" a $\left(\mu_{\mathrm{B}} / \mu_{\mathrm{A}}\right)$ entidades da espécie B. Exemplos:

a) Para reações ácido-base: $\mathrm{H}_{3} \mathrm{O}^{+}+\mathrm{OH}^{-} \rightleftharpoons 2 \mathrm{H}_{2} \mathrm{O}$, podemos ter

$\mathrm{NaOH} / \mathrm{HCl}$.......... $\mathrm{f}_{\text {eq }}[\mathrm{HCl}]=1$, ou seja, $\mathrm{n}(\mathrm{HCl})=$
$\mathrm{n}(\mathrm{NaOH}) ;$ $\mathrm{NaOH} / \mathrm{H}_{2} \mathrm{SO}_{4}$ feq $\left[\mathrm{H}_{2} \mathrm{SO}_{4}\right]=1 / 2$, ou seja, $\mathrm{n}(\mathrm{NaOH})=\mathrm{n}\left(1 / 2 \mathrm{H}_{2} \mathrm{SO}_{4}\right)$;

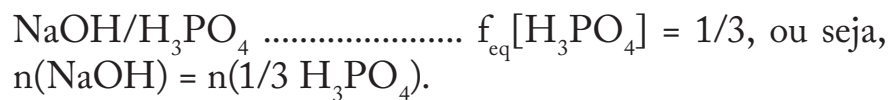
Deve-se notar que no caso de reações de ácidos polipróticos, como o $\mathrm{H}_{3} \mathrm{PO}_{4}$, com bases monohidroxiladas, como 
o $\mathrm{NaOH}$, os valores de feq podem assumir valores fracionários (ex: 1/2 ou 1/3), além do unitário, dependendo da quantidade de base adicionada, em relação ao ácido poliprótico. Isto corresponde à formação majoritária das espécies $\mathrm{H}_{2} \mathrm{PO}_{4}^{-}\left(\mathrm{f}_{\mathrm{eq}}=1\right), \mathrm{HPO}_{4}^{2-}\left(\mathrm{f}_{\mathrm{eq}}=1 / 2\right)$ ou $\mathrm{PO}_{4}^{3-}\left(\mathrm{f}_{\mathrm{eq}}\right.$ $=1 / 3)$. Assim, as concentrações em termos de massal volume de uma solução $1 \mathrm{~N}$ de ácido fosfórico poderiam ser $0,098 \mathrm{~kg} / \mathrm{L}, 0,049 \mathrm{~kg} / \mathrm{L}$ ou $0,033 \mathrm{~kg} / \mathrm{L}$, respectivamente, dependendo do valor de feq. Desse modo, para se usar inequivocamente o termo "normalidade" é preciso explicitar a reação e o fator de equivalência. Assim expressa, a concentração normal é igual à concentração em quantidade de matéria e, portanto, redundante.

b) Para as reações de óxido-redução, o Fator de Equivalência estará associado aos números de elétrons envolvidos nas semi-reações. $\mathrm{Na}$ reação usada para a determinação de ferro,

$$
\begin{gathered}
5 \mathrm{Fe}^{2+} \rightleftharpoons 5 \mathrm{Fe}^{3+}+5 \mathrm{e}^{-} \\
\mathrm{MnO}_{4}^{-}+8 \mathrm{H}^{+}+5 \mathrm{e}^{-} \rightleftharpoons \mathrm{Mn}^{2+}+4 \mathrm{H}_{2} \mathrm{O} \\
5 \mathrm{Fe}^{2+}+\mathrm{MnO}_{4}^{-}+8 \mathrm{H}^{+} \rightleftharpoons 5 \mathrm{Fe}^{3+}+\mathrm{Mn}^{2+}+4 \mathrm{H}_{2} \mathrm{O}
\end{gathered}
$$

O Fator de Equivalência do permanganato de potássio é $\mathrm{f}_{\text {eq }}\left[\mathrm{KMnO}_{4}\right]=1 / 5$, ou seja, $\mathrm{n}\left(\mathrm{Fe}^{2+}\right)=\mathrm{n}\left(1 / 5 \mathrm{MnO}^{4-}\right)$, enquanto que para a reação de padronização do $\mathrm{KMnO}_{4}$ com oxalato de sódio

$\mathrm{C}_{2} \mathrm{O}_{4}{ }^{2-} \rightleftharpoons \mathrm{CO}_{2}+2 \mathrm{e}^{-}(\mathrm{x} 5)$

$\mathrm{MnO}_{4}^{-}+8 \mathrm{H}^{+}+5 \mathrm{e}^{-} \rightleftharpoons \mathrm{Mn}_{2}^{+}+4 \mathrm{H}_{2} \mathrm{O}(\mathrm{x} 2)$

$5 \mathrm{C}_{2} \mathrm{O}_{4}^{2-}+2 \mathrm{MnO}_{4}^{-}+16 \mathrm{H}^{+} \rightleftharpoons 5 \mathrm{CO}_{2}+2 \mathrm{Mn}^{2+}+8 \mathrm{H}_{2} \mathrm{O}$,

o Fator de Equivalência do permanganato de potássio é $\mathrm{f}_{\text {eq }}\left[\mathrm{KMnO}_{4}\right]=2 / 5$, ou seja,

$$
\mathrm{n}\left(\mathrm{C}_{2} \mathrm{O}_{4}^{2-}\right)=\mathrm{n}\left(2 / 5 \mathrm{MnO}^{4-}\right)
$$

de modo que uma mesma espécie poderá apresentar diferentes valores de feq, dependendo da reação.

Assim sendo, a principal restrição à expressão de concentrações em normalidade é o fato do equivalente de uma substância não ser constante e variar de acordo com a reação em que está envolvida. Por conseguinte, recomendase que as concentrações expressas em normalidade sejam abandonadas.
Referências Bibliográficas

1. "The International System of Units", NIST Special Publication 330, National Institute of Standards and Technology, U.S. Department of Commerce, p. 1-55, 1991.

2. "Standard for Use of the International System of Units (SI) - The Modern Metric System”, IEEE/ASTM SI 10-1997, The Institute of Electrical and Electronics Engineers, Inc e American Society for Testing and Materials, p. 1-65, 1997.

3. Cohen, E.R.; Taylor, B.N., "The Fundamental Physical Constants", Physics Today - 10th Annual Buyers' Guide, Part 2, 1993: 9-13.

4. Nelson, R.A., "Guide for Metric Practice", Physics Today - 10th Annual Physics Today Buyers' Guide, Part2, 1993: 15-16.

5. Cantarella, H.; de Andrade, J.C., "O Sistema Internacional de Unidades e a Ciência do Solo", Bol. Inf. Soc. Bras. Ci. Solo, 1992, 17: 91-102

6. Rocha Filho, R.C.; Silva, R.R., "Sobre o Uso Correto de Certas Grandezas em Química”, Quím. Nova, 1991, 14: 300-305. 